\title{
Humoral reaction in the inflamed colon in Hirschsprung's disease and ulcerative colitis
}

\author{
G R ARMSTRONG, F RAAFAT \\ From the Department of Histopathology, The Children's Hospital, Birmingham
}

SUMMARY An immunological study of B cells in patients with colitis that was associated with Hirschsprung's disease was undertaken and compared with that in patients with ulcerative colitis and in normal controls. There was an appreciable increase in IgA and a decrease in IgG in both disease groups. Humoral reaction in colitis associated with Hirschsprung's disease seems to be indistinguishable from that in ulcerative colitis and possibly in other inflammatory bowel diseases.

Despite advances in the rapid and accurate diagnosis of Hirschsprung's disease, in particular the acetylcholinesterase technique, ${ }^{\prime}$ and the availability of effective surgical treatment, it remains a potentially fatal condition.

Severe colitis in Hirschsprung's disease may be the presenting feature, particularly in neonates, ${ }^{2}$ or may occur despite adequate and completed surgical treatment. The clinical and pathological features are those of a necrotising colitis or toxic megacolon, precipitating severe shock and death. Although clearly related to Hirschsprung's disease as a recognised complication, the colitis bears no special relation to the aganglionic segment, and no distinct aetiological agent has been identified. Defined cases can be identified in groups of patients with Hirschsprung's disease and colitis. We recently identified pseudomembranous colitis due to Clostridium difficile as a cause of colitis in some patients with Hirschsprung's disease ${ }^{2}$ but most cases of colitis in Hirschsprung's disease are unexplained. The possibility of an immunological mechanism underlying the colitis has been considered ${ }^{3}$ and the same mechanism may apply in ulcerative colitis, which can present as a life threatening colitis with no apparent aetiological agent. The possible immunological mechanisms underlying chronic inflammatory bowel disease, including the possible roles of immune complexes, cell mediated immunity and local antibody production have been reviewed by Keren. ${ }^{4}$ We looked at local antibody production in Hirschsprung's disease and ulcerative colitis to determine whether there was a difference between the two diseases.

\section{Material and methods}

A group of 11 patients with histologically diagnosed Hirschsprung's disease from this hospital was selected (mean age 14 months; range 9 months to 2 years). Each patient had undergone bowel resection for Hirschsprung's disease. The bowel specimens showed musocal inflammation which could be classified as moderately active chronic colitis (group 1). The criteria were an infiltrate of mononuclear cells in the lamina propria with focal infiltration of crypts by neutrophil polymorphs, with or without crypt abscess formation. Two sections were chosen from each case, a ganglionic segment (group la) and an aganglionic segment (group $1 \mathrm{~b}$ ). In each case the degree of inflammation was similar in the ganglionic and aganglionic segments.

Group 2 comprised 11 patients seen in the same period (1982-85) with a clear diagnosis of ulcerative colitis and a colonic or rectal biopsy specimen showing moderately active chronic inflammation similar to that defined for group 1. The mean age was 7 years 6 months (range 2 to 14 years).

Group 3 comprised 10 patients who had had biopsies of colon or rectum because of a clinical suspicion of Hirschsprung's disease but who had uninflamed bowel with normal ganglion cells. The mean age was 19 months (range 6 months to 3 years).

In all cases, including those finally diagnosed as ulcerative colitis, the diagnosis of Hirschsprung's disease had been established or excluded by suction rectal biopsy and a three step procedure: frozen sections taken to orientate the specimen and identify ganglion cells, followed by histochemical stains to look for increased cholinergic fibres (acetylcholinesterase staining), and lastly, confirmation of the above two steps by serial sections of the paraffin wax embedded biopsy specimen. 
In all three groups a further criterion for inclusion was that a well orientated $5 \mu \mathrm{m}$ section with adequate mucosa was available, to permit examination of three separate high power fields $(\times 400)$.

Immunoglobulins were stained in each group by a standard indirect immunoperoxidase technique using polyclonal antisera against the immunoglobulin subclasses IgG, IgA, IgM, and IgE (Dakopatts) with appropriate positive and negative controls. The stained sections were identified by the departmental accession numbers and were examined randomly. They were not classified into groups 1-3 until morphometrical analysis was completed.

The immmunoperoxidase stained sections were analysed with a Kontron MOP 30 image analyser. A high power field from a Leitz projector microscope was projected on to the measuring grid of the analyser so that one high power field included the full thickness of well orientated mucosa in most of the sections.

For each section, three separate high power fields were chosen. The area of lamina propria included in the field was measured and all of the cells in the three high power fields that showed cytoplasmic staining for each immunoglobulin subclass were counted. From these data the number of immunoglobulin containing plasma cells in each square millimetre of lamina propria was calculated.

\section{Results}

All of the sections in each case were comparable in the orientation of mucosa and the amount that was counted. All cells with clearly positive cytoplasmic staining were counted and most had the morphological characteristics of lymphocytes or plasma cells. Only a few cells stained positively for IgE, and these were excluded from the study. The results in each case were expressed for each immunoglobulin class as a percentage of the total number of positive cells of all classes per square millimetre of lamina propria in each case (table).

The mean number of IgM containing cells was identical in all three groups (Hirschsprung's disease,

Table Number (SD) of cells staining positively for each immunoglobulin subclass as a percentage of total number of stained cells per square millimetre of lamina propria

\begin{tabular}{llll}
\hline Group & $I g A$ & $I g M$ & $I g G$ \\
\hline la & $63 \cdot 8$ & $11 \cdot 9$ & $24 \cdot 4$ \\
& $(18 \cdot 96)$ & $(3.67)$ & $(16.73)$ \\
lb & $62 \cdot 5$ & $18 \cdot 3$ & $19 \cdot 1$ \\
& $(18 \cdot 29)$ & $(10 \cdot 12)$ & $(9 \cdot 36)$ \\
& $65 \cdot 0$ & $12 \cdot 1$ & $22 \cdot 9$ \\
& $(15 \cdot 90)$ & $(10 \cdot 02)$ & $(11 \cdot 71)$ \\
& $42 \cdot 8$ & $12 \cdot 9$ & $44 \cdot 3$ \\
& $(18 \cdot 89)$ & $(8 \cdot 43)$ & $(17 \cdot 03)$ \\
\hline
\end{tabular}

ulcerative colitis, and controls); the IgA and IgG containing cells were more and less numerous, respectively, in the disease groups than in the control colonic biopsy specimens.

Student's $t$ test was used for comparative analysis. For IgA containing cells significant differences were observed between groups $1 \mathrm{a}$ and $3(\mathrm{p} 0.02)$, groups $1 \mathrm{~b}$ and 3 (p 0.05), and groups 2 and 3 (p 0.05). For IgG containing cells, significant differences were observed between groups $1 \mathrm{a}$ and 3 (p 0.02), groups $1 \mathrm{~b}$ and 3 (p 0.001 ), and groups 2 and 3 (p 0.01). No significant differences were observed for either IgA or IgG when comparing group $1 \mathrm{a}$ and group 2 , group $1 \mathrm{~b}$ and group 2 , or group la and group $1 \mathrm{~b}$. There was no significant difference between any groups for IgM.

\section{Discussion}

Several reports on the immunopathology of ulcerative colitis and Crohn's disease have been published. Previous reports suggest that there is an increase in IgG positive cells in inflamed bowels compared with normal colons and this is most clearly seen when the colitic group is divided into quiescent and active disease. Keren compared the intracellular immunoglobulin content of the lamina propria with disease activity in ulcerative colitis and showed an increase in intracellular IgG, IgA, and IgM in active inflammation, but noted that the IgG tended to be focally increased and the $\operatorname{IgA}$ and IgM diffusely increased. ${ }^{5}$ Other workers, however, produced different results. Rosenkrause et al showed that there was an increase in IgM in Crohn's disease, suggesting that this was a differentiating feature between Crohn's disease and ulcerative colitis. ${ }^{6}$ O'Donoghue and Kumar showed an increase in IgE in ulcerative colitis and Crohn's disease and suggested an allergic aetiology. ${ }^{7}$ This rise in IgE probably represents a small group of patients with a specific probability for allergic conditions. Schweider et al attempted to differentiate between ulcerative colitis and Crohn's disease by immunohistological analysis and showed an increase in $\operatorname{IgA}$ and IgG in active disease, with IgA showing the greater increase. ${ }^{8}$ They also looked at complement components. Scott $e t$ al showed a general increase in immunoglobulin and could not distinguish between different types of inflammatory bowel disease by this method. ${ }^{9}$

Fewer studies have concentrated on local antibody production in Hirschsprung's disease. Wijesinha and Steer looked at the dysfunctional bowel in children with necrotising colitis and Hirschsprung's disease who had had a proximal colostomy, and compared the number of immunoglobulin containing cells in the proximal and distal colon. ${ }^{10}$ They showed that the distal bowel had fewer positive cells of IgA and IgM subclasses and they related this to an absence of 
luminal antigens in the dysfunctional bowel. In a different study the same authors showed an increase in IgG and a decrease in IgA containing cells in obstructed (ganglionic) segments compared with aganglionic segments in a small group of patients with Hirschsprung's disease. ${ }^{3}$ Their results also suggest that the numbers of positive cells return to normal with relief of obstruction. There might, therefore, be an immunological response to a luminal antigen and an IgG mediated response similar to that described in ulcerative colitis. Halpin et al suggested an immunological component in Hirschsprung's disease when they measured IgG in explants of the colon in the newborn infants 1 : a raised IgG value was found in Hirschsprung's disease and not in other types of obstructive lower intestinal disease. As the mean age of their patients was 3.5 days this immunoglobulin was presumed to be at least partly maternally derived.

Our results show a different pattern from that seen in other studies. In particular, we have shown that there is an increase of IgA containing cells but not of IgG containing cells in the Hirschsprung's disease and colitis groups. This may be related to the degree of inflammation, as other studies have found increased mucosal IgG containing cells in severe inflammation ${ }^{56}$ but our groups were chosen to show only moderate degrees of inflammation (the commonest type in untreated Hirschsprung's disease). Furthermore, their studies of inflammatory bowel disease were in adults and our group were all children.

While these differences make comparison with other studies difficult, the important finding is that there is a fairly clear difference between our two disease groups and the controls, but no difference between the two disease groups themselves. Schweider suggested counting plasma cells as a means of distinguishing between inflammatory bowel diseases of different aetiologies. ${ }^{8}$ With the exception of the rare IgE mediated allergic proctitis, however, our results and those of other workers using commercial antisera' show that this is of no help in individual cases because of the wide scatter of results within groups. We suggest that the assessment of local antibody response does not help in the differentiation of the two commonest inflammatory diseases of the colon, and does not distinguish between the inflammation associated with Hirschsprung's disease and that seen in ulcerative colitis.

We thank Mr Martyn Peck for technical assistance and Miss Marna Aitken for typing the manuscript.

\section{References}

1 Meier-Ruge W. Hirschsprung's disease. In: Holschneider AM, ed. Morphological diagnosis. New York: Thieme Stratton, 1982:6271.

2 Brearley S, Armstrong GR, Nairn R, et al. Pseudomembranous colitis-a lethal complication of Hirschsprung's disease unrelated to antibiotic usage. J Paediatr Surg 1987;22:257-9.

3 Wijesinha SS, Steer HW. Observations on the immunocytes and macrophages in megacolon. Dis Colon Rectum 1982;25:312-20.

4 Keren DF. Immunopathogenesis of inflammatory bowel disease. In: Thomas Norris, ed. Pathology of the colon, small intestine and anus. London: Churchill Livingstone, 1983:61-76.

5 Keren DF, Appelman HD, Dobbins WO, et al. Correlation of histopathologic evidence of disease activity with the presence of immunoglobulin-containing cells in the colon of patients with inflammatory bowel disease. Hum Pathol 1984;15:757-63.

6 Rosenkraus PCM, Meijer CJLM, Van Der Wal AM, Cornelisse CJ, Lindeman J. Immunoglobulin containing cells in inflammatory bowel disease of the colon: a morphometric and immunohistochemical study. Gut 1980;21:941-7.

7 O'Donoghue DP, Kumer P. Rectal IgE cells in inflammatory bowel disease. Gut 1979;20:149-53.

8 Schneider HM, Loos M, Storkel S, Gross M. Immunohistological differential diagnosis of inflammatory colonic diseases. Histopathology 1984;8:583-8.

9 Scott BB, Goodall A, Stephenson P, Jenkins D. Rectal mucosal plasma cells in inflammatory bowel disease. Gut 1983;24:51924.

10 Wijesinha SS, Steer HW. Studies of the immunoglobulin-producing cells of the human intestine: the defunctional bowel. Gut 1982;23:211-14.

11 Halpin TC, Gregoire RP, Izant RJ. Abnormal rectal immunoglobulin pattern in Hirschsprung's disease. Lancet 1978; ii:606-8.

Requests for reprints to: Dr F Raafat, Department of Histopathology, Birmingham Children's Hospital, Ladywood Middleway, Birmingham B16 8ET, England. 